\section{OPEN ACCESS}

Edited by:

Jose Vilela-Martin

Faculdade de Medicina de São José

do Rio Preto, Brazil

Reviewed by:

Yi Zhang,

Tongji University, China Maria Lorenza Muiesan,

University of Brescia, Italy

*Correspondence:

Weiliang Wang

docwangwl@hotmail.com

Junli Zuo

zuo-junli@163.com

†These authors have contributed equally to this work

Specialty section This article was submitted to

Hypertension

a section of the journal

Frontiers in Cardiovascular Medicine

Received: 15 October 2021

Accepted: 04 January 2022

Published: 22 February 2022

Citation:

Hu Y, Zhao J, Wang Q, Chao H,

Tang B, Cheng D, Tan I, Butlin M, Avolio A, Wang W and Zuo J (2022) Disparate Associations of 24-h Central

Aortic and Brachial Cuff Blood

Pressure With Hypertension-Mediated Organ Damage and Cardiovascular

Front. Cardiovasc. Med. 9:795509.

doi: 10.3389/fcvm.2022.795509

\title{
Disparate Associations of 24-h Central Aortic and Brachial Cuff Blood Pressure With Hypertension-Mediated Organ Damage and Cardiovascular Risk
}

Yueliang Hu ${ }^{1 t}$, Jiehui Zhao ${ }^{2 \dagger}$, Qian Wang ${ }^{1+}$, Huijuan Chao ${ }^{1}$, Biwen Tang ${ }^{1}$, Di Cheng ${ }^{1}$, Isabella Tan ${ }^{3}$, Mark Butlin ${ }^{3}$, Alberto Avolio ${ }^{3}$, Weiliang Wang ${ }^{4 *}$ and Junli Zuo ${ }^{1,3 *}$

\footnotetext{
'Department of Geriatrics, Ruijin Hospital, Shanghai Jiao Tong University School of Medicine, Shanghai, China, ${ }^{2}$ Daning Community Health Service Center, Shanghai, China, ${ }^{3}$ Macquarie Medical School, Faculty of Medicine, Health and Human Sciences, Macquarie University, Sydney, NSW, Australia, ${ }^{4}$ Xuhui Center Hospital, Shanghai, China
}

Objective: Aim of this study was to evaluate the associations of non-invasive central aortic and peripheral (brachial) blood pressure (BP) for Hypertension-mediated organ damage $(\mathrm{HMOD})$ and atherosclerotic cardiovascular disease (ASCVD) risk.

Methods: We evaluated associations of HMOD with 24-h ambulatory blood pressure monitoring (ABPM) of central aortic and peripheral BP indices in patients with primary hypertension and presence of several cardiovascular risk factors. BP measurements were performed by means of a non-invasive automated oscillometric device (Mobil-O-Graph). HMOD was defined as the presence of carotid intima-media thickness (IMT) above normal values and/or carotid plaque, left ventricular hypertrophy $(\mathrm{LVH})$, and/or renal abnormalities as assessed by urine albumin/creatinine ratio above normal values and/or estimated glomerular filtration rate (eGFR) $<60 \mathrm{ml} / \mathrm{min}$ per $1.73 \mathrm{~m}^{2}$.

Results: In the study cohort of 273 (age $55.2 \pm 13.4$ years, $71.8 \%$ male) patients with primary hypertension, documented HMOD was present in 180 (65.9\%), LVH in 70 (25.6\%), increased IMT in 129 (47.3\%). Fifty-six patients (20.5\%) had kidney organ damage (20.5\% albuminuria and $2.6 \%$ impaired eGFR). When accounting for confounding factors (age, sex, body-mass-index, antihypertensive treatment, smoking, triacylglycerol, statin treatment, glucose, hypoglycemic therapy, or heart rate) only peripheral 24-h pulse pressure (PP) maintained statistical significance with HMOD indices (OR: 1.126, 95\% Cl: 1.012 1.253; $p=0.029$ ). Using ASCVD risk score as the independent continuous variable in multiple linear regression, 24-h central systolic pressure (SBP) ( $\beta=0.179 ; 95 \%$ Cl:0.019 0.387; $p=0.031$ ), daytime central PP ( $\beta=0.114 ; 95 \% \mathrm{Cl}: 0.070 \sim 0.375 ; p=0.005$, night-time central SBP $(\beta=0.411 ; 95 \% \mathrm{Cl}: 0.112 \sim 0.691 ; \beta=0.007)$ and night-time $\mathrm{PP}(\beta=0.257$; 95\% Cl:0.165 0.780; $p=0.003)$ were all positively associated with ASCVD risk. 


\begin{abstract}
Conclusions: Blood pressure obtained by 24-h ABPM was better correlated with HMOD than office BP. Whilst 24-h peripheral BP showed a stronger association with HMOD than 24-h central BP, the prognostic value of 24-h central BP for the 10-year ASCVD risk was superior to 24-h peripheral BP.
\end{abstract}

Keywords: central blood pressure, peripheral blood pressure, atherosclerotic cardiovascular disease, hypertension-mediated organ damage, non-invasive haemodynamics indices

\section{INTRODUCTION}

With the change of lifestyle, the total number of hypertension patients in China has reached the alarming number of 245 million (1). As the most important risk factor for cardiovascular disease, hypertension has brought us a serious burden of disease. Statistics show that the prevalence and mortality of cardiovascular diseases in the Chinese are still on the rise. In 2017, 2.54 million people in China died due to high blood pressure (BP), of which $95.7 \%$ died of cardiovascular disease (2). Interventions for hypertension, especially early intervention, are urgently needed.

Changes in large arteries and microcirculation play a role in the mechanism of BP and HMOD (hypertension-mediated organ damage) (3). The increase in arterial stiffness caused by aging and other factors lead to an increase in systolic BP (SBP). The increase in SBP and pulse pressure (PP) transmit to the distal vascular system, leading to microcirculation damage, remodeling, and related endothelial dysfunction. Eventually, target organs such as heart, brain and kidney and blood vessels themselves are damaged.

HMOD is related to increased vascular risk and death, and their prevention should become the target of hypertension treatment and a surrogate sign of whether BP is wellcontrolled (4). It is theorized that at the time of diagnosis, all adult hypertension patients have HMOD, but the severity varies. Cardiovascular death, myocardial infarction, stroke, endstage renal disease (ESRD) or heart failure are common and irreversible late complications (4). For young hypertensive patients, it is more important to prevent the development of HMOD.

The Chinese Hypertension Guidelines have pointed out that assessing HMOD is an important part of the diagnosis and evaluation of hypertension. After early detection and treatment, asymptomatic subclinical HMOD can be reversed, which helps to improve the prognosis of patients (5).

In this study, we investigated the use different indicators to evaluate the correlation between different BP indices and HMOD such as heart, kidney, and large blood vessel damage, hoping to clearly distinguish the intervention targets for early detection, intervention and reversal of HMOD.

\section{MATERIALS AND METHODS \\ Study Population}

In this cross-sectional study between July 2018 and July 2020, 273 (196 men, age $55.21 \pm 13.37$ years) inpatients enrolled in the Department of Geriatrics, Shanghai Ruijin Hospital,
China, who suffered from primary hypertension with presence of several cardiovascular risk factors or complications involving clinical HMOD were recruited. The inclusion criteria were 3 consecutive measurements of office SBP $\geq 140 \mathrm{mmHg}$ or consultation diastolic $\mathrm{BP}(\mathrm{DBP}) \geq 90 \mathrm{mmHg}$ after resting for $5 \mathrm{~min}$ in the supine position, or the use of antihypertensive drugs without the diagnosis of secondary hypertension. Exclusion criteria were clinical or laboratory evidence confirming the acute cardiocerebrovascular disease within the previous 3 months before enrollment or any life-threatening disease.

Patients' medical history, smoking habits, medication history, and auxiliary examinations [serum total cholesterol (TC), highdensity lipoprotein cholesterol (HDL-C), low-density lipoprotein cholesterol (LDL-C), triglycerides (TG), blood glucose, serum creatinine, and urine albumin/creatinine ratio (ACR)] were collected in the form of standardized questionnaires. Cardiovascular risk was determined according to the American College of Cardiology/American Heart AssociationAtherosclerotic Cardiovascular Disease (ACC/AHA-ASCVD) risk score (6).

Current smoking was defined as having smoked in their lifetime or smoked in the past 7 days of BP measurement $(7,8)$. Diabetes mellitus was defined as a fasting blood glucose concentration of at least $7 \mathrm{mmol} / \mathrm{l}$, or a random blood glucose level of over $11.1 \mathrm{mmol} / \mathrm{l}$, or the use of antidiabetic drugs. Dyslipidemia was defined as serum TC $\geq 240 \mathrm{mg} / \mathrm{dl}$, LDL-C $\geq 160 \mathrm{mg} / \mathrm{dl}$, HDL-C $\leq 35 \mathrm{mg} / \mathrm{dl}$, TG $\geq 150 \mathrm{mg} / \mathrm{dl}$, or the use of antilipidemic drugs according to Adult Treatment Panel III cardiovascular risk factor definitions (9). The study protocol was reviewed and approved by the Ethics Committee of Ruijin Hospital, Shanghai Jiaotong University School of Medicine. All patients provided written informed consent.

\section{Blood Pressure Measurements}

Both office and 24-h BP measurements were performed by means of a non-invasive automated oscillometric device (Mobil-O-Graph PWV; IEM, Stolberg, Germany), validated for brachial BP measurement, according to the European Society of Hypertension International protocol (10).

The monitor was fitted on a weekday between 8:00 am and 8:00 am. After 5-min rest, BP was measured four times consecutively at 1-min intervals. The mean of these measurements was settled as office(clinic) BP. The device was set to function under the manufacturer's inbuilt protocol number 11, namely: four BP recordings per hour from 0800 to $2,359 \mathrm{~h}$ and two recordings per hour from 0000 to $0759 \mathrm{~h}$. In this mode of function, the device performs both brachial BP measurement 
and brachial pressure wave recording simultaneously. The aortic (central) BP is assessed by software analysis [with the application of validated generalized transfer functions $(11,12)]$ after the data are downloaded to the manufacturer's software (HMS version 4.6). The aortic BP derived by the Mobil-O-Graph NG apparatus has been compared vs. the non-invasive "gold standard" apparatus for central BP estimation (Sphygmocor device) in four studies (12-17), as well as vs. the invasive aortic BP recordings in one study (13), with consistently quite acceptable results $(11,14)$. The feasibility and/or reproducibility of the device to assess central hemodynamics, both in large populations at rest (17) and during ambulatory monitoring $(15,18)$, has been also evaluated with satisfactory results.

All patients included in the study had recordings of good technical quality (at least $80 \%$ of valid readings). Otherwise, ABPM was repeated in 1 week.

PPA was defined as the peripheral-to-central pulse pressure ratio and was calculated using the formula: $\mathrm{PPA}=$ (brachial SBPbrachial DBP)/(central SBP - central DBP).

\section{HMOD Indices}

HMOD was defined as the presence of carotid intimamedia thickness (IMT) above normal values $(0.9 \mathrm{~mm})$ and/or carotid plaque, left ventricular hypertrophy $(\mathrm{LVH})$, and/or renal abnormalities as assessed by urine albumin/creatinine ratio (ACR) above normal values $(>3.5 \mathrm{mg} / \mathrm{mmol}$ in females and $>2.5$ $\mathrm{mg} / \mathrm{mmol}$ in males) and/or an estimated glomerular filtration rate $(\mathrm{eGFR})<60 \mathrm{ml} / \mathrm{min}$ per $1.73 \mathrm{~m}^{2}$.

\section{Intima-Media Thickness}

We used high-resolution echocardiography Doppler ultrasound (HD11EX Ultrasound; Philips Medical Systems, Andover, MA, USA) with a broadband linear array transducer (multiple frequency: 4-12 MHz) to examine Carotid IMT.IMT was measured on both the left and right common carotid artery starting $\sim 1.5 \mathrm{~cm}$ proximal to the carotid artery bulb. During the test, three records were collected from the left and right carotid arteries, and the average value of each side was calculated. When IMT $>1.3 \mathrm{~mm}$, carotid plaque was diagnosed. When IMT $\geq 0.9 \mathrm{~mm}$ and/or carotid plaque was present, carotid artery abnormality was diagnosed.

\section{Left Ventricular Hypertrophy}

$\mathrm{LVH}$ was defined as a left ventricular mass index (LVMI) $\geq$ $115 \mathrm{~g} / \mathrm{m}^{2}$ in men or $\geq 95 \mathrm{~g} / \mathrm{m}^{2}$ in women and was calculated by means of echocardiography, performed according to the American Society of Echocardiography recommendations (19).

\section{Renal Abnormalities}

The definition and the diagnostic criteria for chronic kidney disease (CKD) were proposed in the Kidney Disease Outcomes Quality Initiative (K/DOQI) guidelines: (eGFR $<60 \mathrm{ml} / \mathrm{min} / 1.73$ $\mathrm{m}^{2}$ ) as calculated by the MDRD formula (20) or urinary ACR > $3.5 \mathrm{mg} / \mathrm{mmol}$ in females and $>2.5 \mathrm{mg} / \mathrm{mmol}$ in males were used to screen hypertensive patients with CKD.

\section{The Atherosclerotic Cardiovascular Disease (ASCVD) Risk}

The ACC/AHA-ASCVD risk score was determined using values of TC, HDL-C, LDL-C, age, BP, gender, presence of diabetes, and smoking status (6). The ACC/AHA-ASCVD risk score was defined as high risk when the score $\geq 7.5 \%$ and low risk when score $<7.5 \%(21)$.

\section{Statistical Analysis}

We use SPSS 24.0 for Windows (SPSS Inc., Chicago, IL, USA) for statistical analysis. When $p<0.05$ on both sides, it is considered statistically significant.

Continuous variables are expressed as mean $\pm \mathrm{SD}$, and frequencies (percentage) are reported for categorical variables. Student's $t$-test or Chi-square test were used for the comparison between patients with and without HMOD. Pearson correlation was used to assess the relations between brachial, central aortic BP and LVMI, ACR, eGFR, or IMT. The association of each BP estimate with the presence of HMOD was assessed by means of logistic regression analyses, with odds ratio (OD) calculation adjusted for age, sex, body mass index (BMI), antihypertensive treatment, smoking, TG, statin treatment, fasting glucose, glucose, hypoglycemic therapy, heart rate.

Furthermore, the relative impact of central vs. peripheral BP estimates was assessed by the simultaneous introduction of each pair of central and peripheral measurements (office, 24-h, daytime, and night-time) in the logistic regression models, and then calculating the adjusted ODs. The association of each BP estimate with the presence of ASCVD risk was assessed by means of linear regression with adjustment for baseline covariates including age, sex, BMI, antihypertensive treatment, smoking, TG, statin treatment, fasting glucose, glucose, hypoglycemic therapy, heart rate.

\section{RESULTS}

\section{Characteristics of Participants}

The study cohort comprised $273(71.8 \%$ men) patients with primary hypertension. A total of $180(65.9 \%)$ patients had documented HMOD. LVH was present in 70 patients $(25.6 \%)$, increased IMT in 129 (47.3\%). A total of 56 patients $(20.5 \%)$ had kidney organ damage (20.5\% albuminuria and 2.6\% impaired eGFR). From them, 66 patients $(24.2 \%)$ presented HMOD in more than one organ.

Table 1 shows the difference in clinical parameters and BP measurements between patients with and without HMOD. Patients with HMOD had significantly higher brachial and aortic (central) BP, measured during 24-h, day or night except clinic peripheral PP and clinic aortic PP. Patients with HMOD were older, more frequently treated, and more with associated glucose disorders. As expected, values of serum creatinine, ACR, LVMI, IMT, and ASCVD risk score were increased and eGFR reduced in the group with HMOD. The PPA was higher in HMOD group, but there was no statistical significance.

Table 2 summarizes the correlations between peripheral and central $\mathrm{BP}$ and HMOD indices. There were different strengths of correlation between BP and HMOD indices. LVMI was 
TABLE 1 | Characteristics of the study subjects.

\begin{tabular}{|c|c|c|c|c|}
\hline Characteristics & $\begin{array}{c}\text { Total } \\
(n=273)\end{array}$ & $\begin{array}{l}\text { Without HMOD } \\
\qquad(n=93)\end{array}$ & $\begin{array}{l}\text { With HMOD } \\
\qquad(n=180)\end{array}$ & $P$-value \\
\hline Age,y & $55.21 \pm 13.37$ & $50.10 \pm 12.59$ & $57.82 \pm 13.02$ & $<0.001$ \\
\hline Sex (\% men) & 28.2 & 28.9 & 26.9 & 0.727 \\
\hline $\mathrm{BMI} /\left(\mathrm{kg} / \mathrm{m}^{2}\right)$ & $26.49 \pm 5.09$ & $26.32 \pm 5.53$ & $26.57 \pm 4.87$ & 0.701 \\
\hline Antihypertensive treatment (\%) & 83.5 & 75.3 & 87.8 & 0.008 \\
\hline Glucose (mmol/L) & $5.92 \pm 1.59$ & $5.5 \pm 1.10$ & $6.14 \pm 1.76$ & 0.002 \\
\hline Triglycerides (mmol/L) & $2.06 \pm 1.97$ & $2.09 \pm 1.29$ & $2.04 \pm 2.24$ & 0.854 \\
\hline Total cholesterol (mmol/L) & $4.73 \pm 0.98$ & $4.80 \pm 0.78$ & $4.69 \pm 1.07$ & 0.384 \\
\hline LDL-C (mmol/L) & $3.13 \pm 0.75$ & $3.19 \pm 0.60$ & $3.09 \pm 0.82$ & 0.320 \\
\hline Creatinine $(\mu \mathrm{mol} / \mathrm{L})$ & $78.59 \pm 15.50$ & $77.10 \pm 12.11$ & $79.36 \pm 16.96$ & 0.256 \\
\hline ASCVD(\%) & $15.00 \pm 13.07$ & $9.54 \pm 8.63$ & $17.60 \pm 14.01$ & $<0.001$ \\
\hline eGFR(ml/min per $\left.1.73 \mathrm{~m}^{2}\right)$ & $90.24 \pm 15.53$ & $93.19 \pm 13.50$ & $88.71 \pm 16.31$ & 0.024 \\
\hline LVMI $\left(\mathrm{g} / \mathrm{m}^{2}\right)$ & $98.18 \pm 23.09$ & $86.47 \pm 13.68$ & $104.23 \pm 24.62$ & $<0.001$ \\
\hline IMT (mm) & $0.73 \pm 0.10$ & $0.69 \pm 0.07$ & $0.75 \pm 0.11$ & $<0.001$ \\
\hline LgACR (mg/mmol) & $0.43 \pm 0.45$ & $0.25 \pm 0.24$ & $0.53 \pm 0.50$ & $<0.001$ \\
\hline Clinic Peripheral SBP & $133.17 \pm 18.45$ & $129.76 \pm 18.01$ & $134.94 \pm 18.44$ & 0.029 \\
\hline Clinic Aortic SBP & $123.81 \pm 16.90$ & $120.78 \pm 15.60$ & $125.38 \pm 17.37$ & 0.044 \\
\hline Clinic Peripheral PP & $44.86 \pm 13.39$ & $42.72 \pm 12.93$ & $45.97 \pm 13.52$ & 0.059 \\
\hline Clinic Aortic PP & $33.72 \pm 10.90$ & $32.41 \pm 10.51$ & $34.41 \pm 11.07$ & 0.176 \\
\hline Clinic PPA & $1.32 \pm 0.17$ & $1.31 \pm 0.13$ & $1.33 \pm 0.19$ & 0.380 \\
\hline 24-h Peripheral SBP & $126.69 \pm 11.94$ & $122.76 \pm 11.41$ & $128.74 \pm 11.72$ & $<0.001$ \\
\hline 24-h Aortic SBP & $118.11 \pm 11.05$ & $115.46 \pm 11.30$ & $119.51 \pm 10.69$ & 0.004 \\
\hline 24-h Peripheral PP & $43.00 \pm 9.27$ & $40.35 \pm 7.79$ & $44.38 \pm 9.70$ & 0.001 \\
\hline 24-h Aortic PP & $33.07 \pm 7.09$ & $31.87 \pm 7.00$ & $33.71 \pm 7.08$ & 0.043 \\
\hline 24-h PPA & $1.31 \pm 0.12$ & $1.29 \pm 0.07$ & $1.32 \pm 0.15$ & 0.051 \\
\hline Daytime Peripheral SBP & $128.06 \pm 12.06$ & $124.41 \pm 12.03$ & $129.97 \pm 11.67$ & $<0.001$ \\
\hline Daytime Aortic SBP & $119.09 \pm 11.07$ & $116.17 \pm 10.84$ & $120.62 \pm 10.90$ & 0.002 \\
\hline Daytime Peripheral PP & $42.79 \pm 9.05$ & $40.58 \pm 8.02$ & $43.94 \pm 9.35$ & 0.004 \\
\hline Daytime Aortic PP & $32.33 \pm 6.86$ & $30.94 \pm 6.00$ & $33.07 \pm 7.18$ & 0.015 \\
\hline Daytime PPA & $1.33 \pm 0.10$ & $1.31 \pm 0.08$ & $1.34 \pm 0.10$ & 0.081 \\
\hline Night-time Peripheral SBP & $122.95 \pm 14.52$ & $117.66 \pm 12.27$ & $125.66 \pm 14.86$ & $<0.001$ \\
\hline Night-time Aortic SBP & $115.43 \pm 13.12$ & $111.05 \pm 11.32$ & $117.67 \pm 13.44$ & $<0.001$ \\
\hline Night-time Peripheral PP & $42.86 \pm 9.92$ & $39.93 \pm 8.57$ & $44.35 \pm 10.25$ & $<0.001$ \\
\hline Night-time Aortic PP & $34.20 \pm 7.34$ & $32.4 \pm 7.03$ & $35.14 \pm 7.35$ & 0.004 \\
\hline Night-time PPA & $1.25 \pm 0.12$ & $1.24 \pm 0.09$ & $1.26 \pm 0.13$ & 0.127 \\
\hline
\end{tabular}

Data are mean $\pm S D$ or percentage as marked. P-value: independent t-test analysis of variance for numeric variables and chi-square test for categoric variables.

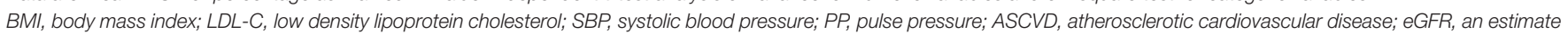
of GFR for the modified MDRD formula; ACR, Urine albumin-to-creatinine; PPA, peripheral-to-central pulse pressure ratio.

significantly associated with both brachial and central SBP parameters for clinic, 24-h, day and night $(r=0.121-0.281, p<$ $0.001)$. ACR was significantly associated with both brachial and central SBP as well as PP parameters for clinic,24-h, day and night $(r=0.151-0.299, p<0.01)$ except clinic aortic SBP and clinic aortic PP. It was also significantly associated with Clinic PPA and Night-time PPA $(r=0.147-0.148, p<0.05)$ except 24 -h PPA and Daytime PPA.There was a significant negative association between eGFR and the brachial and central PP for 24-h, day and night $(r=-0.205$ to $-0.234, p<0.001)$ except night-time aortic PP. It was also significantly associated with Clinic PPA and Night-time PPA ( $r=-0.134$ to $-0.144, p<0.05$ ) except 24 $\mathrm{h}$ PPA and Daytime PPA.IMT was significantly associated with both brachial and central PP parameters for 24-h, day and night $(r=0.141-0.163, p<0.05)$.

Table 3 shows adjusted ODs for office and ambulatory BP estimates. When each pair of BP value (central and peripheral) were introduced in the same logistic regression model, only peripheral BP estimates (24-h pPP) maintained their statistical significance, whereas none of the central BP parameters were significant after adjustment for age, sex, BMI, antihypertensive treatment, smoking, TG, statin treatment, glucose, hypoglycemic therapy, heart rate (Figure 1).

When PPA (Clinic, 24-h PPA, Daytime, Night-time) were introduced in the same logistic regression model, none of PPA parameters were significant after adjustment for age, sex, 
TABLE 2 | Relationship between BP and HMOD.

\begin{tabular}{|c|c|c|c|c|c|c|c|c|}
\hline \multirow[t]{2}{*}{ Variable } & \multicolumn{2}{|c|}{ LVMI } & \multicolumn{2}{|c|}{$\log A C R$} & \multicolumn{2}{|c|}{ eGFR } & \multicolumn{2}{|c|}{ IMT } \\
\hline & $r$-value & $P$-value & $r$ value & $P$-value & $r$-value & $P$-value & $r$-value & $P$-value \\
\hline Age,y & 0.065 & 0.287 & 0.109 & 0.072 & $-0.404^{\star \star}$ & $<0.001$ & $0.350^{\star \star}$ & $<0.001$ \\
\hline Sex & $-0.225^{\star \star}$ & $<0.001$ & 0.096 & 0.113 & $-0.176^{\star \star}$ & 0.004 & -0.079 & 0.208 \\
\hline $\mathrm{BMI} /\left(\mathrm{kg} / \mathrm{m}^{2}\right)$ & 0.105 & 0.086 & -0.070 & 0.251 & 0.086 & 0.158 & 0.037 & 0.554 \\
\hline Glucose (mmol/L) & 0.070 & 0.264 & $0.286^{\star \star}$ & $<0.001$ & -0.060 & 0.341 & 0.125 & 0.052 \\
\hline Triglyceride (mmol/L) & $0.194^{\star \star}$ & 0.001 & 0.020 & 0.737 & -0.021 & 0.736 & $-0.213^{\star \star}$ & 0.001 \\
\hline Total cholesterol (mmol/L) & $-0.165^{\star \star}$ & 0.006 & 0.006 & 0.925 & $-0.121^{\star}$ & 0.047 & -0.061 & 0.331 \\
\hline LDL-C (mmol/L) & $-0.157^{\star \star}$ & 0.010 & 0.017 & 0.780 & -0.113 & 0.063 & -0.045 & 0.475 \\
\hline Clinic Peripheral SBP & $0.236^{\star \star}$ & $<0.001$ & $0.161^{*}$ & 0.008 & 0.024 & 0.700 & 0.079 & 0.212 \\
\hline Clinic Aortic SBP & $0.255^{\star \star}$ & $<0.001$ & 0.111 & 0.083 & 0.071 & 0.271 & 0.048 & 0.472 \\
\hline Clinic Peripheral PP & 0.111 & 0.069 & $0.161^{\star \star}$ & 0.008 & -0.113 & 0.064 & 0.101 & 0.108 \\
\hline Clinic Aortic PP & 0.088 & 0.173 & 0.066 & 0.308 & -0.063 & 0.329 & 0.079 & 0.233 \\
\hline Clinic PPA & 0.115 & 0.073 & $0.147^{\star}$ & 0.022 & $-0.134^{*}$ & $0.037^{\star}$ & 0.049 & 0.461 \\
\hline 24-h Peripheral SBP & $0.281^{\star \star}$ & $<0.001$ & $0.299^{\star \star}$ & $<0.001$ & -0.058 & 0.343 & 0.096 & 0.127 \\
\hline 24-h Aortic SBP & $0.252^{\star \star}$ & $<0.001$ & $0.151^{*}$ & 0.013 & 0.005 & 0.939 & 0.110 & 0.081 \\
\hline 24-h Peripheral PP & 0.100 & 0.100 & $0.286^{\star \star}$ & $<0.001$ & $-0.234^{\star \star}$ & $<0.001$ & $0.157^{\star}$ & 0.012 \\
\hline 24-h Aortic PP & 0.079 & 0.194 & $0.237^{\star \star}$ & $<0.001$ & $-0.205^{\star \star}$ & 0.001 & $0.163^{\star \star}$ & 0.009 \\
\hline 24-h PPA & 0.073 & 0.230 & 0.043 & 0.477 & -0.060 & 0.328 & 0.042 & 0.506 \\
\hline Daytime Peripheral SBP & $0.265^{\star \star}$ & $<0.001$ & $0.280^{\star \star}$ & $<0.001$ & -0.047 & 0.439 & 0.085 & 0.176 \\
\hline Daytime Aortic SBP & $0.254^{\star \star}$ & $<0.001$ & $0.232^{\star \star}$ & $<0.001$ & 0.006 & 0.924 & 0.064 & 0.312 \\
\hline Daytime Peripheral PP & 0.077 & 0.208 & $0.279^{\star \star}$ & $<0.001$ & $-0.229^{\star \star}$ & $<0.001$ & $0.141^{*}$ & 0.025 \\
\hline Daytime Aortic PP & 0.056 & 0.363 & $0.258^{\star \star}$ & $<0.001$ & $-0.225^{\star \star}$ & $<0.001$ & $0.153^{\star}$ & 0.015 \\
\hline Daytime PPA & 0.074 & 0.223 & 0.062 & 0.308 & -0.004 & 0.954 & -0.027 & 0.664 \\
\hline Night-time Peripheral SBP & $0.253^{\star \star}$ & $<0.001$ & $0.297^{\star \star}$ & $<0.001$ & -0.075 & 0.220 & 0.109 & 0.082 \\
\hline Night-time Aortic SBP & $0.255^{\star \star}$ & $<0.001$ & $0.250^{\star \star}$ & $<0.001$ & -0.015 & 0.808 & 0.099 & 0.118 \\
\hline Night-time Peripheral PP & $0.121^{*}$ & 0.048 & $0.277^{\star \star}$ & $<0.001$ & $-0.208^{\star \star}$ & 0.001 & $0.148^{*}$ & 0.018 \\
\hline Night-time Aortic PP & 0.078 & 0.202 & $0.155^{\star}$ & 0.011 & -0.111 & 0.072 & $0.154^{\star}$ & 0.014 \\
\hline Night-time PPA & 0.033 & 0.596 & $0.148^{*}$ & 0.016 & $-0.144^{\star}$ & 0.019 & -0.014 & 0.827 \\
\hline
\end{tabular}

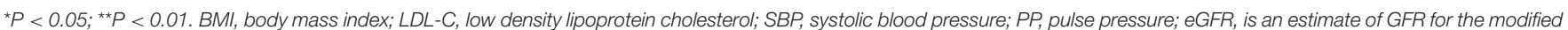
MDRD formula.

BMI, antihypertensive treatment, smoking, TG, statin treatment, glucose, hypoglycemic therapy, heart rate.

The left ventricular mass was further used as an independent HMOT index and analyzed as the independent continuous variable in multiple linear regression, we found that 24$\mathrm{h}$ peripheral SBP was more relevant to LVMI than 24-h central SBP $(\beta=0.161 ; 95 \%$ CI:0.064 0.562; $p=0.014)$ after adjustment for age, sex, BMI, antihypertensive treatment, smoking, TG, statin treatment, glucose, hypoglycemic therapy, heart rate. Night-time central SBP is more relevant to LVMI than night-time peripheral SBP $(\beta=0.134 ; 95 \%$ CI:0.008 0.473; $p=0.042$ ) when accounting for confounding factors, Using ASCVD risk score as the independent continuous variable in multiple linear regression, central but not peripheral 24-h SBP, daytime PP, night-time SBP and PP were all positively associated with ASCVD risk after adjusted for traditional cardiovascular risk factors (Table 4). In addition, Daytime PPA( $\beta$ $=-0.096 ; 95 \% \mathrm{CI}:-0.238$ to $-0.036 .387 ; p=0.008)$ and Night-time PPA ( $\beta=-0.098$; 95\% CI: -0.193 to $-0.030 ; p$ $=0.007)$ were all negatively ssociated with ASCVD risk after adjusted for confounding factors except Clinic PPA and 24h PPA.

\section{DISCUSSION}

ABPM data of HMOD patients showed significantly higher BP compared to patients without HMOD, but the clinic (office) BP did not increase significantly. This suggested that even for patients with normal clinic BP, we need to be vigilant and realize that clinic BP does not provide complete information.

Although HMOD and non-HMOD patients had no difference in clinic peripheral and central PP and SBP, HMOD patients showed significantly higher BP levels on multiple indicators during ABPM. Furthermore, only central but not peripheral BP was associated with increased 10-year ASCVD risk.

BP measurement is a fundamental means and method for evaluating BP levels, diagnosing hypertension, and observing the efficacy of antihypertensive treatment. In clinical and population prevention work, the main use of office BP 
TABLE 3 | Odds ratios and 95\% confidence intervals for each mmHg increase of the association of each blood pressure value with the presence of Hypertension-mediated organ damage.

\begin{tabular}{|c|c|c|c|c|c|c|}
\hline & Model 1 & Model 2 & Model 3 & Model 4 & Model 5 & Model 6 \\
\hline Clinic Peripheral SBP & $1(0.935 \sim 1.07)$ & $0.995(0.929 \sim 1.065)$ & $0.997(0.931 \sim 1.067)$ & $0.999(0.933 \sim 1.07)$ & $1(0.929 \sim 1.076)$ & $0.950(0.873 \sim 1.033)$ \\
\hline Clinic Aortic SBP & 1.025 (0.952 1.103) & $1.028(0.954 \sim 1.108)$ & $1.026(0.952 \sim 1.105)$ & $1.024(0.95 \sim 1.103)$ & $1.026(0.947 \sim 1.112)$ & 1.085 (0.989 1.190) \\
\hline Clinic Peripheral PP & 1.022 (0.961 1.086) & $1.012(0.951 \sim 1.077)$ & $1.014(0.953 \sim 1.079)$ & $1.016(0.954 \sim 1.082)$ & 1.02 (0.955 1.091) & $0.976(0.901 \sim 1.058)$ \\
\hline Clinic Aortic PP & $0.985(0.915 \sim 1.06)$ & $0.996(0.924 \sim 1.073)$ & $0.995(0.923 \sim 1.073)$ & $0.993(0.921 \sim 1.071)$ & $0.993(0.916 \sim 1.076)$ & $1.047(0.947 \sim 1.157)$ \\
\hline 24-h pSBP & $1.095(1.013 \sim 1.184)$ & $1.092(1.01 \sim 1.181)$ & 1.092 (1.01 1.182) & $1.092(1.009 \sim 1.182)$ & $1.11(1.011 \sim 1.218)$ & $1.095(0.999 \sim 1.200)$ \\
\hline 24-h cSBP & $0.951(0.876 \sim 1.033)$ & $0.95(0.874 \sim 1.032)$ & $0.949(0.873 \sim 1.032)$ & $0.95(0.873 \sim 1.033)$ & $0.937(0.849 \sim 1.035)$ & $0.949(0.862 \sim 1.046)$ \\
\hline 24-h pPP & $1.121(1.017 \sim 1.235)$ & $1.114(1.015 \sim 1.223)$ & 1.115 (1.015 1.224) & $1.116(1.016 \sim 1.227)$ & $1.136(1.026 \sim 1.257)$ & $1.126(1.012 \sim 1.253)$ \\
\hline 24-h cPP & $0.894(0.791 \sim 1.01)$ & $0.897(0.798 \sim 1.009)$ & 0.897 (0.798 1.009) & $0.896(0.796 \sim 1.009)$ & $0.885(0.778 \sim 1.006)$ & $0.882(0.771 \sim 1.009)$ \\
\hline Daytime pSBP & $1.073(0.964 \sim 1.195)$ & $1.068(0.959 \sim 1.188)$ & $1.068(0.959 \sim 1.188)$ & $1.067(0.958 \sim 1.189)$ & $1.086(0.97 \sim 1.215)$ & $1.066(0.945 \sim 1.203)$ \\
\hline Daytime cSBP & $0.97(0.864 \sim 1.09)$ & $0.972(0.865 \sim 1.091)$ & $0.971(0.865 \sim 1.091)$ & $0.972(0.865 \sim 1.093)$ & $0.958(0.848 \sim 1.082)$ & $0.976(0.856 \sim 1.113)$ \\
\hline Daytime pPP & $1.11(1.009 \sim 1.222)$ & $1.104(1.003 \sim 1.215)$ & $1.104(1.004 \sim 1.215)$ & $1.108(1.005 \sim 1.22)$ & 1.12 (1.013 1.239) & $1.098(0.986 \sim 1.223)$ \\
\hline Daytime cPP & $0.896(0.791 \sim 1.015)$ & $0.9(0.794 \sim 1.019)$ & $0.9(0.794 \sim 1.019)$ & $0.896(0.79 \sim 1.017)$ & $0.888(0.777 \sim 1.015)$ & $0.902(0.780 \sim 1.043)$ \\
\hline Night-time pSBP & $1.021(0.936 \sim 1.114)$ & $1.022(0.937 \sim 1.116)$ & $1.023(0.937 \sim 1.117)$ & $1.025(0.938 \sim 1.12)$ & $1.053(0.962 \sim 1.153)$ & $1.047(0.951 \sim 1.154)$ \\
\hline Night-time cSBP & $1.024(0.932 \sim 1.125)$ & $1.019(0.927 \sim 1.12)$ & $1.018(0.926 \sim 1.12)$ & $1.016(0.924 \sim 1.118)$ & $0.991(0.9 \sim 1.092)$ & $0.993(0.895 \sim 1.100)$ \\
\hline Night-time pPP & $1.042(0.96 \sim 1.131)$ & $1.038(0.957 \sim 1.127)$ & 1.039 (0.957 1.127) & $1.041(0.958 \sim 1.13)$ & 1.067 (0.981 1.16) & $1.054(0.963 \sim 1.154)$ \\
\hline Night-time cPP & $0.985(0.89 \sim 1.09)$ & $0.987(0.893 \sim 1.092)$ & $0.987(0.892 \sim 1.092)$ & $0.985(0.89 \sim 1.09)$ & $0.963(0.869 \sim 1.066)$ & $0.969(0.868 \sim 1.082)$ \\
\hline
\end{tabular}

pSBP, Peripheral systolic blood pressure; cSBP, Aortic systolic blood pressure; pPP, Peripheral pulse pressure; cPP, Aortic pulse pressure.

Model 1 adjusting for Age,Sex, BMI.

Model 2 adjusting for Age,Sex, BMI, Antihypertensive treatment.

Model3 adjusting for Age, Sex, BMI, Antihypertensive treatment, Smoker.

Model 4 adjusting for Age, Sex, BMI, Antihypertensive treatment,Smoker, Triacylglycerol.

Model 5 adjusting for Age, Sex, BMI, Antihypertensive treatment,Smoker, Triacylglycerol, Statin treatment.

Model 6 adjusting for Age, Sex, BMI, Antihypertensive treatment, Smoker, Triacylglycerol, Statin treatment, Glucose, Hypoglycemic therapy, HR.

The bold values represent the values that are statistically significant $(P<0.05)$.

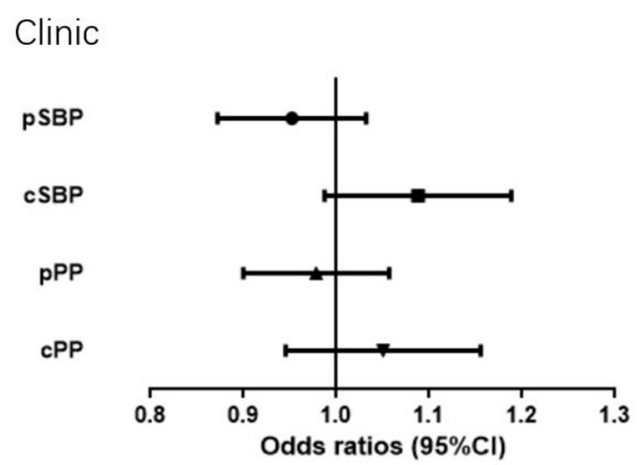

$24 \mathrm{H}$

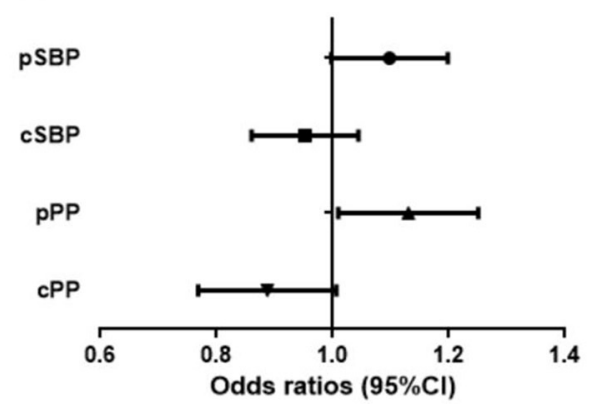

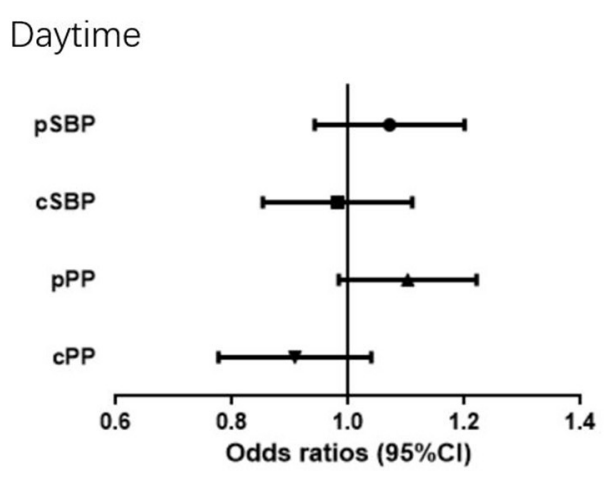

Nighttime

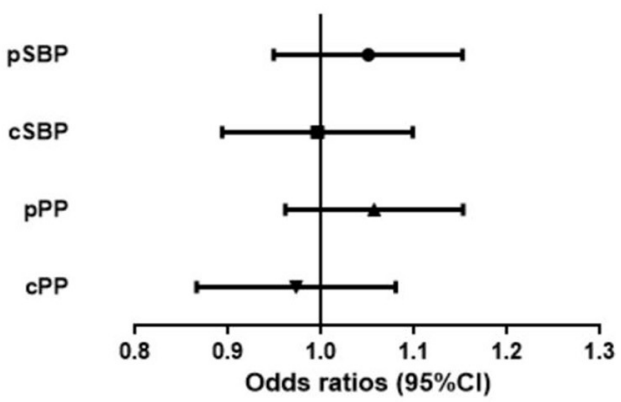

FIGURE 1 | Odds ratio (OR) and 95\% Confidence Interval (Cl) of Hypertension-mediated organ damage with the all BP measurements. 
TABLE 4 | Linear regression analysis of associations between each blood pressure value and ASCVD.

\begin{tabular}{|c|c|c|c|c|c|}
\hline \multirow[b]{2}{*}{ Variable } & \multirow[b]{2}{*}{ B } & \multicolumn{4}{|c|}{ ASCVD (\%) } \\
\hline & & SE & $\beta$ & $P$-value & $95 \% \mathrm{Cl}$ \\
\hline \multicolumn{6}{|l|}{ Model 1} \\
\hline 24-h pSBP (mmHg) & 0.167 & 0.166 & 0.153 & 0.152 & $-0.062 \sim 0.395$ \\
\hline 24-h cSBP (mmHg) & 0.158 & 0.124 & 0.136 & 0.204 & $-0.087 \sim 0.403$ \\
\hline 24-h pPP & 0.047 & 0.121 & 0.032 & 0.701 & $-0.192 \sim 0.285$ \\
\hline 24-h cPP & 0.341 & 0.149 & 0.182 & $0.023^{\star}$ & $0.048 \sim 0.634$ \\
\hline Daytime pSBP & -0.05 & 0.231 & -0.046 & 0.83 & $-0.506 \sim 0.406$ \\
\hline Daytime cSBP & 0.376 & 0.25 & 0.321 & 0.135 & $-0.117 \sim 0.87$ \\
\hline Daytime pPP & -0.141 & 0.207 & -0.092 & 0.498 & $-0.549 \sim 0.267$ \\
\hline Daytime cPP & 0.592 & 0.266 & 0.3 & $0.027^{*}$ & $0.066 \sim 1.117$ \\
\hline Night-time pSBP & -0.148 & 0.171 & -0.167 & 0.386 & $-0.485 \sim 0.188$ \\
\hline Night-time cSBP & 0.43 & 0.185 & 0.437 & $0.021^{*}$ & $0.065 \sim 0.796$ \\
\hline Night-time pPP & -0.053 & 0.16 & -0.038 & 0.741 & $-0.368 \sim 0.263$ \\
\hline Night-time cPP & 0.393 & 0.2 & 0.216 & 0.051 & $-0.001 \sim 0.786$ \\
\hline \multicolumn{6}{|l|}{ Model 2} \\
\hline 24-h pSBP (mmHg) & 0.141 & 0.113 & 0.13 & 0.214 & $-0.082 \sim 0.365$ \\
\hline 24-h cSBP (mmHg) & 0.139 & 0.122 & 0.119 & 0.254 & $-0.101 \sim 0.378$ \\
\hline 24-h pPP & 0.082 & 0.116 & 0.056 & 0.483 & $-0.148 \sim 0.311$ \\
\hline 24-h cPP & 0.258 & 0.144 & 0.138 & 0.074 & $-0.025 \sim 0.542$ \\
\hline Daytime pSBP & -0.072 & 0.226 & -0.067 & 0.75 & $-0.516 \sim 0.373$ \\
\hline Daytime cSBP & 0.356 & 0.244 & 0.304 & 0.146 & $-0.125 \sim 0.837$ \\
\hline Daytime pPP & -0.146 & 0.199 & -0.096 & 0.465 & $-0.538 \sim 0.246$ \\
\hline Daytime cPP & 0.556 & 0.256 & 0.282 & $0.031^{*}$ & $0.051 \sim 1.06$ \\
\hline Night-time pSBP & -0.145 & 0.167 & -0.163 & 0.388 & $-0.475 \sim 0.185$ \\
\hline Night-time cSBP & 0.384 & 0.182 & 0.39 & $0.036^{\star}$ & $0.026 \sim 0.743$ \\
\hline Night-time pPP & -0.086 & 0.154 & -0.062 & 0.578 & $-0.389 \sim 0.218$ \\
\hline Night-time cPP & 0.376 & 0.192 & 0.207 & 0.051 & $-0.002 \sim 0.754$ \\
\hline \multicolumn{6}{|l|}{ Model 3} \\
\hline 24-h pSBP (mmHg) & 0.127 & 0.104 & 0.117 & 0.224 & $-0.078 \sim 0.331$ \\
\hline 24-h cSBP (mmHg) & 0.138 & 0.111 & 0.119 & 0.215 & $-0.081 \sim 0.358$ \\
\hline 24-h pPP & 0.07 & 0.106 & 0.048 & 0.509 & $-0.139 \sim 0.279$ \\
\hline 24-h cPP & 0.296 & 0.131 & 0.158 & $0.025^{\star}$ & $0.038 \sim 0.555$ \\
\hline Daytime pSBP & -0.155 & 0.207 & -0.143 & 0.454 & $-0.562 \sim 0.252$ \\
\hline Daytime cSBP & 0.431 & 0.224 & 0.367 & 0.055 & $-0.01 \sim 0.871$ \\
\hline Daytime pPP & -0.208 & 0.181 & -0.137 & 0.252 & $-0.566 \sim 0.149$ \\
\hline Daytime cPP & 0.641 & 0.233 & 0.326 & $0.006^{*}$ & $0.181 \sim 1.101$ \\
\hline Night-time pSBP & -0.092 & 0.155 & -0.104 & 0.551 & $-0.397 \sim 0.212$ \\
\hline Night-time cSBP & 0.309 & 0.168 & 0.314 & 0.068 & $-0.023 \sim 0.64$ \\
\hline Night-time pPP & -0.078 & 0.14 & -0.056 & 0.575 & $-0.354 \sim 0.197$ \\
\hline Night-time cPP & 0.374 & 0.174 & 0.206 & $0.033^{\star}$ & $0.031 \sim 0.718$ \\
\hline \multicolumn{6}{|l|}{ Model4 } \\
\hline 24-h pSBP (mmHg) & 0.127 & 0.104 & 0.117 & 0.226 & $-0.079 \sim 0.332$ \\
\hline 24-h cSBP (mmHg) & 0.137 & 0.112 & 0.118 & 0.221 & $-0.083 \sim 0.357$ \\
\hline 24-h pPP & 0.067 & 0.106 & 0.046 & 0.527 & $-0.142 \sim 0.276$ \\
\hline 24-h cPP & 0.299 & 0.131 & 0.16 & $0.024^{\star}$ & $0.041 \sim 0.558$ \\
\hline Daytime pSBP & -0.162 & 0.207 & -0.15 & 0.437 & $-0.57 \sim 0.247$ \\
\hline Daytime cSBP & 0.436 & 0.224 & 0.372 & 0.053 & $-0.006 \sim 0.878$ \\
\hline Daytime pPP & -0.236 & 0.183 & -0.155 & 0.198 & $-0.596 \sim 0.124$ \\
\hline Daytime cPP & 0.676 & 0.235 & 0.344 & $0.004^{\star}$ & $0.213 \sim 1.14$ \\
\hline Night-time pSBP & -0.102 & 0.156 & -0.115 & 0.512 & $-0.409 \sim 0.204$ \\
\hline
\end{tabular}

(Continued)
TABLE 4 | Continued

\begin{tabular}{|c|c|c|c|c|c|}
\hline \multirow[b]{2}{*}{ Variable } & \multirow[b]{2}{*}{ B } & \multicolumn{4}{|c|}{ ASCVD (\%) } \\
\hline & & SE & $\beta$ & $P$-value & 95\% Cl \\
\hline Night-time cSBP & 0.316 & 0.169 & 0.321 & 0.062 & $-0.017 \sim 0.649$ \\
\hline Night-time pPP & -0.084 & 0.14 & -0.061 & 0.55 & $-0.361 \sim 0.193$ \\
\hline Night-time cPP & 0.379 & 0.175 & 0.208 & $0.031^{*}$ & $0.035 \sim 0.723$ \\
\hline \multicolumn{6}{|l|}{ Model 5} \\
\hline 24-h pSBP (mmHg) & 0.127 & 0.104 & 0.117 & 0.225 & $-0.078 \sim 0.332$ \\
\hline 24-h cSBP (mmHg) & 0.135 & 0.112 & 0.116 & 0.227 & $-0.085 \sim 0.356$ \\
\hline 24-h pPP & 0.053 & 0.106 & 0.036 & 0.617 & $-0.157 \sim 0.263$ \\
\hline 24-h cPP & 0.315 & 0.132 & 0.168 & $0.018^{\star}$ & $0.055 \sim 0.574$ \\
\hline Daytime pSBP & -0.166 & 0.207 & -0.154 & 0.423 & $-0.575 \sim 0.242$ \\
\hline Daytime cSBP & 0.44 & 0.224 & 0.375 & 0.051 & $-0.002 \sim 0.881$ \\
\hline Daytime pPP & -0.246 & 0.183 & -0.161 & 0.18 & $-0.605 \sim 0.114$ \\
\hline aytime cPP & 0.688 & 0.235 & 0.349 & $0.004^{\star}$ & $0.225 \sim 1.151$ \\
\hline Night-time pSBP & -0.087 & 0.157 & -0.098 & 0.579 & $-0.397 \sim 0.222$ \\
\hline Night-time cSBP & 0.299 & 0.17 & 0.304 & 0.081 & $-0.037 \sim 0.635$ \\
\hline Night-time pPP & -0.074 & 0.142 & -0.053 & 0.601 & $-0.354 \sim 0.205$ \\
\hline Night-time cPP & 0.366 & 0.177 & 0.201 & $0.039^{\star}$ & $0.018 \sim 0.714$ \\
\hline \multicolumn{6}{|l|}{ Model 6} \\
\hline 24-h pSBP (mmHg) & -0.008 & 0.088 & -0.007 & 0.931 & $-0.181 \sim 0.166$ \\
\hline 24-h cSBP (mmHg) & 0.203 & 0.093 & 0.179 & $0.031^{*}$ & $0.019 \sim 0.387$ \\
\hline 24-h pPP & 0.026 & 0.088 & 0.018 & 0.771 & $-0.148 \sim 0.199$ \\
\hline 24-h cPP & 0.15 & 0.11 & 0.081 & 0.175 & $-0.067 \sim 0.366$ \\
\hline Daytime pSBP & -0.349 & 0.17 & -0.334 & 0.042 & $-0.685 \sim-0.013$ \\
\hline Daytime cSBP & 0.572 & 0.184 & 0.506 & $0.002^{\star}$ & $0.209 \sim 0.936$ \\
\hline Daytime pPP & 0.107 & 0.06 & 0.071 & 0.076 & $-0.011 \sim 0.226$ \\
\hline Daytime cPP & 0.222 & 0.077 & 0.114 & $0.005^{\star}$ & $0.070 \sim 0.375$ \\
\hline Night-time pSBP & -0.263 & 0.139 & -0.294 & 0.059 & $-0.537 \sim 0.010$ \\
\hline Night-time cSBP & 0.402 & 0.147 & 0.411 & $0.007^{\star}$ & $0.112 \sim 0.691$ \\
\hline Night-time pPP & -0.241 & 0.13 & -0.170 & 0.064 & $-0.497 \sim 0.015$ \\
\hline Night-time cPP & 0.472 & 0.156 & 0.257 & $0.003^{*}$ & $0.165 \sim 0.780$ \\
\hline
\end{tabular}

pSBP, Peripheral systolic blood pressure; cSBP, Aortic systolic blood pressure; pPP, Peripheral pulse pressure; CPP, Aortic pulse pressure.

Model 1 adjusting for Age, Sex, BMI.

Model 2 adjusting for Age, Sex, BMI, Antihypertensive treatment.

Model 3 adjusting for Age, Sex, BMI, Antihypertensive treatment, Smoker.

Model 4 adjusting for Age, Sex, BMI, Antihypertensive treatment, Smoker, Triacylglycerol. Model 5 adjusting for Age, Sex, BMI, Antihypertensive treatment, Smoker, Triacylglycerol, Statin treatment.

Model 6 adjusting for Age, Sex, BMI, Antihypertensive treatment, Smoker, Triacylglycerol, Statin treatment, Glucose, Hypoglycemic therapy, HR.

The symbol * and bold values represent the values that are statistically significant $(P<0.05)$.

measurement and out-of-office BP measurement, the latter includes $\mathrm{ABPM}$ and home BP monitoring (HBPM), can provide a large amount of BP data outside the medical environment (5).

Clinic BP, recommend by most clinical guidelines (22), has been considered as the basis for guiding therapeutic decisions focused on the cardiovascular protection (23), closely related to prognosis. However, in the last two decades, ABPM, developed initially to study the circadian changes in $\mathrm{BP}$ and to determine the influence of BP-lowering drugs on the 24-h BP profile (24), has become important in determining BP of individuals, as it 
provides a more accurate prognosis and a better association with HMOD in hypertensive patients with respect to clinic BP (2528). In our study, compared to clinic BP, BP obtained during 24-h ABPM had a stronger correlation with HMOD, which suggests it could be routinely used for assessment of hypertensive patients with suspected HMOD.

We found that different BP indicators have different correlations with HMOD: SBP and PP are better correlated with ACR, PP is better correlated with eGFR and IMT, and SBP is better correlated with LVMI.

The increase of SBP will increase the vascular pressure at the end of systole, and the increase of PP will cause vascular endothelial damage and mechanical fatigue, leading to atherosclerosis. Kong MG et al. showed SBP and PP are better HMOD indicators (29).It showed patients undergoing elective invasive coronary angiography (CAG), SBP, and PP had stronger relationships with E/e' and coronary artery disease than DBP and mean arterial pressure (MAP).

Jokiniitty et al. (30) suggested that PP is the most significant BP indicator in predicting future LVMI and change in LVMI. Cirillo et al. (31) suggested that in non-diabetic, middle-aged adults, PP and isolated systolic hypertension are directly related to microalbuminuria. The South Korean study (29) suggested that neither SBP nor PP was related to LVMI and eGFR. A study by Chinese investigators (32) suggested that SBP and severe hypertension (grade 3) were common independent risk factors for HMOD. These differences may be related to sample size, BP level, and age of study cohort.

In general, $\mathrm{PP}$ is an independent risk factor for cardiovascular disease and mortality, and there is a clear link between PP and HMOD (29). In addition, the influence of SBP and overall BP levels cannot be ignored.

It is well-known that SBP and PP are higher when assessed at the brachial artery compared to the aorta because of PP amplification across the arterial tree (33). Therefore, target organs, especially the heart and large arteries, which are directly exposed to central rather than brachial BP, could be more strongly affected by the former (33). In fact, central BP has been increasingly considered to be a better estimator than the traditional peripheral BP measurement, found to be more closely related with HMOD, such as LV mass, carotid intima-media thickening, and pulse wave velocity (34). What is more, as shown by some longitudinal studies, central BP has a better predictive value of future cardiovascular events and mortality in hypertensive patients (35-38).

In most of the studies showing better predictive value of central BP, central and peripheral BP estimates were taken at clinic measurements of BP. In this study we investigated if ABPM might give the same result: whether it be assumed that 24-h central arterial pressure is superior to peripheral arterial pressure in predicting HMOD and long-term prognosis. However, seemingly contradictory results have been obtained in this study. The 24-h central BP did indeed show a greater predictive value for the 10-year ASCVD risk than 24-h peripheral BP. But when it comes to correlation with HMOD, the opposite is true: 24 -h peripheral BP showed a stronger association with HMOD than 24-h central BP.This result is consistent with the findings of Spanish investigators (39).

The conclusion appears paradoxical. Why is the central ABPM better than the peripheral in 10-year CV risk but poorer in HMODs? In fact, in the past few years, research in this area has consistently shown conflicting results. Studies have suggested that the correlation between central $\mathrm{BP}$ and cardiovascular events is better than peripheral BP $(35,36)$. Other studies, like ours, have not found the advantage of central $\mathrm{BP}(37,38)$ specific to HMOD, but rather for ASCVD. The difference in measurement and correction methods may be the reason for the different conclusions reached in different studies. In addition, we think the more critical point is that patients were mostly treated and this might have significantly influenced the results. After early detection and timely treatment, subclinical HMOD can be reversed (40). In contrast, the adverse association between cardiovascular outcomes and aortic sclerosis cannot be reversed by antihypertensive therapy, which may be one of the apparent reasons for the contradictory results of this study.

The finding of a lack of association with $24 \mathrm{~h}$ brachial BP and IMT is surprising and differs from several other findings in the general population (41) or in hypertensive patients (42). We postulate that the difference may be related to the following factors. Most of the selected population received antihypertensive therapy in our study, while the patients in the other two studies had no history of hypertension or were in the drug washout period. The effect of antihypertensive medication on patients' SBP and PP may be the main reason for the difference. In addition, the measurement method of IMT is different. Our IMT measurement is only for one carotid site, while the other two studies carried out $6 \sim 12$ carotid sites. Further research in a larger sample size of the population may provide further clarification.

Our research results also show that if LVMI is analyzed as a stand-alone HMOD, 24-h peripheral SBP is more relevant to LVMI than 24-h central SBP. Night-time central SBP is more relevant to LVMI than night-time peripheral SBP. This is also contrary to what is generally accepted. It is generally believed that, compared with peripheral blood pressure, central $\mathrm{BP}$ is more closely correlated with LVMI (43). The paradox found in our study is most likely related to changes in antihypertensive treatment and arterial stiffness. Central SBP and PWV parameters are significantly correlated to hypertension (43). To address this issue, we are planning to analyze different PWV phenotypes in patients with hypertension.

Our study also showed that the clinic PPA, 24-h 24PPA, day PPA, night-time PPA had no prognostic value for HMOD. As the day PPA increased, the ASCVD risk decreased, while the Night-time PPA increased, the ASCVD risk decreased. 24-h PPA and clinic PPA had no prognostic value for ASCVD risk. In other words, PPA only has a prognostic effect on ASCVD risk, and has no correlation with HMOD. The Northern Shanghai Study (44) showed that PPA is a cardiac-related biomarker in community-based elderly. It seems understandable that, PPA is an indicator of cardiac pressure load, and it is related to cfPWV. As a vascular biomarker, cfPWV reflects the vessel elasticity of the aorta and is significantly associated with arterial changes, including glomerulus damage (44). PPA is only related to cardiac 
HMOD, so PPA and cfPWV have different prognostic values for HMOD, but they have similar values in ASCVD risk.

Due to the hypervagal function, night-time SBP decreased significantly (>10\%) so the day time peripheral PP was slightly higher than the night-time peripheral PP. Since the PPA of night time is less than the day time, day time SBP does not decrease significantly, resulting in a slightly higher night-time central PP than the day time central PP. Since the relation between central and peripheral PP is highly dependent on heart rate, is expected that changes in heart rate would result in different degree of amplification of PP from the aorta to the periphery.

In future research, we will address the PPA effect of ABPM at different times, especially the prognostic value of nighttime changes to the risk of HMOD and ASCVD.

In this study, HMOD was defined as the presence of carotid IMT above normal values $(0.9 \mathrm{~mm}), \mathrm{LVH}$, and/or renal abnormalities as assessed by urine ACR above normal values ( $>3.5 \mathrm{mg} / \mathrm{mmol}$ in females and $>2.5 \mathrm{mg} / \mathrm{mmol}$ in males) and/or an eGFR $<60 \mathrm{ml} / \mathrm{min}$ per $1.73 \mathrm{~m}^{2}$.

Carotid artery plaque was classified as abnormal carotid artery in this study, and ABI and PWV measurements were not considered to be a manifestation of target organ damage. We adopt this definition of target organ damage to refer to the definition in the 2018 Chinese guidelines for the management of hypertension (40). It was a relatively simple and convenient method for detecting target organ damage. The predictive effect of $\mathrm{ABI}$ and $\mathrm{PWV}$ on patients' cardiovascular risk has been reported in many studies, but at present in China, it is still an investigative indicator rather than a clinical indicator, so we did not include these two indicators for analysis. We will conduct further analysis in future research.

\section{Limitations}

This study has some limitations. As a cross-sectional study with a small sample size, the results need to be further confirmed in prospective studies. The cohort of this study is a physical examination population and the risk factors such as antihypertensive treatment werenot collected, therefore it is unknown whether results are applicable for other disease populations. The study was conducted in an Asian population, and it is not known whether the results will hold true for other ethnic groups.

\section{CONCLUSION}

Blood pressure obtained by 24-h peripheral ABPM was better correlated with HMOD than office BP and 24-h central BP. However, the prognostic value of 24-h central BP for the 10-year ASCVD risk was superior to 24-h peripheral BP.

\section{DATA AVAILABILITY STATEMENT}

The data that support the findings of this study are available from the corresponding author, [Jl Z], upon reasonable request.

\section{ETHICS STATEMENT}

Written informed consent was obtained from the individual(s) for the publication of any potentially identifiable images or data included in this article.

\section{AUTHOR CONTRIBUTIONS}

$\mathrm{YH}$ contributed to the concept and design of the study, the analysis and interpretation of data, and drafting and critically revising the manuscript. QW, JZ, HC, and BT were responsible for study design and analysis and interpretation of data. DC was responsible for the figure. IT contributed to interpretation of data and critically revising the manuscript. $\mathrm{MB}$ and $\mathrm{AA}$ contributed to the study design, the thorough reading of the manuscript and several revisions. JlZ and WW contributed to the conception and design of the study, interpretation of the data, and critically revising the manuscript. All authors approved the final version for publication and agree to be accountable for all aspects of the work in ensuring that questions related to the accuracy or integrity of any part of the work are appropriately investigated and resolved.

\section{FUNDING}

Project Supported by the National Natural Science Foundation of China (Grant No. 81500190), and Clinical Science and Shanghai municipal hospital new frontier technology joint project (SHDC12019X20), Shanghai Municipal Commission of Health and Family Planning (Grant No. 2020YJZX0124).

\section{ACKNOWLEDGMENTS}

We gratefully acknowledge the invaluable assistance of the physicians of the Department of Geriatric Medicine Ruijin Hospital, Shanghai Jiaotong University School of Medicine; the study would not have been possible without their support.

\section{REFERENCES}

1. The Writing Committee of the Report on Cardiovascular Health and Diseases in China. Report on Cardiovascular health and diseases in China 2019: an updated summary. Chin Circul J. (2020) 35:833. doi: 10.3969/j.issn.1000-3614.2020.09.001

2. Zhou M, Wang H, Zeng X, Yin P, Zhu J, Chen W, et al. Mortality, morbidity, and risk factors in China and its provinces, 1990-2017: a systematic analysis

for the Global Burden of Disease Study 2017. Lancet. (2019) 394:114558. doi: 10.1016/S0140-6736(19)30427-1

3. Schiffrin EL. How structure, mechanics, and function of the vasculature contribute to blood pressure elevation in hypertension. Can J Cardiol. (2020) 36:648-58. doi: 10.1016/j.cjca.2020. 02.003

4. Piskorz D. Hypertensive mediated organ damage and hypertension management. how to assess beneficial effects of antihypertensive 
treatments? High Blood Press Cardiovasc Prev. (2020) 27:917. doi: 10.1007/s40292-020-00361-6

5. Writing Group of 2018 Chinese Guidelines for the Management of Hypertension, Chinese Hypertension League, Chinese Society of Cardiology, et al. 2018 Chinese guidelines for the management of hypertension. Chin J Cardiovasc Med. (2019) 24:24-56. doi: 10.3969/j.issn.1007-5410.2019.01.001

6. Goff DC, Lloyd-Jones DM, Bennett G, Coady S, D'Agostino RB, Gibbons R, et al. 2013 ACC/AHA guideline on the assessment of cardiovascular risk: a report of the American College of Cardiology/American Heart Association task force on practice guidelines. J Am Coll Cardiol. (2014) 63:2935-59. doi: 10.1161/01.cir.0000437741.48606.98

7. Tam J, Day HR, Rostron BL, Apelberg BJ. A systematic review of transitions between cigarette and smokeless tobacco product use in the United States. BMC Public Health. (2015) 15:258. doi: 10.1186/s12889-015-1594-8

8. Wetter DW, McClure JB, De Moor C, Cofta-Gunn L, Cummings S, Cinciripini $\mathrm{PM}$, et al. Concomitant use of cigarettes and smokeless tobacco: prevalence, correlates, and predictors of tobacco cessation. Prev Med. (2002) 34:63848. doi: 10.1006/pmed.2002.1032

9. National Cholesterol Education Program (NCEP) Expert Panel on Detection, Evaluation and the T of HBCA (Adult TPI). Third report of the national cholesterol education program (NCEP) expert panel on detection, evaluation, and treatment of high blood cholesterol in adults (Adult treatment panel III) final report. Circulation. (2002) 106:3143-421. doi: 10.1161/circ.106.25.3143

10. Franssen PM, Imholz BP. Evaluation of the Mobil-O-Graph new generation ABPM device using the ESH criteria. Blood Press Monit. (2010) 15:22931. doi: 10.1097/MBP.0b013e328339be38

11. Wassertheurer S, Mayer CC, Breitencker F. Modeling arterial and left ventricular coupling from noninvasive measurements. Simulat Model Theory Pract. (2008) 16:988-97. doi: 10.1016/j.simpat.2008.04.016

12. Wassertheurer S, Kropf J, Weber T, van der Giet M, Baulmann J, Ammer $\mathrm{M}$, et al. A new oscillometric method for pulse wave analysis: comparison with a common tonometric method. J Hum Hypertens. (2010) 24:498504. doi: $10.1038 /$ jhh.2010.27

13. Weber $T$, Wassertheurer S, Rammer $M$, Maurer E, Hametner B, Mayer CC, et al. Validation of a brachial cuff-based method for estimating central systolic blood pressure. Hypertension. (2011) 58:825-32. doi: 10.1161/HYPERTENSIONAHA.111.176313

14. Wilkinson IB, Mc Eniery CN, Cockcroft JR. Central blood pressure estimation for the masses moves a step closer. J Hum Hypertens. (2010) 24:4957. doi: $10.1038 / \mathrm{jhh} .2010 .47$

15. Luzardo L, Lujambio I, Sottolano M, da Rosa A, Thijs L, Noboa O, et al. 24-h ambulatory recording of aortic pulse wave velocity and central systolic augmentation: a feasibility study. Hypertens Res. (2012) 35:9807. doi: $10.1038 / \mathrm{hr} .2012 .78$

16. Weis W, Gohlisch C, Harsch-Gladisch C, Tolle M, Zidek W, van der Giet M. Oscillometric estimation of central blood pressure: validation of the MobilO-Graph in comparison with Sphygmocor device. Blood Press Monit. (2012) 17:128-31. doi: 10.1097/MBP.0b013e328353ff63

17. Nunan D, Wassertheurer S, Lasserson D, Hametner B, Fleming S, Ward A, et al. Assessment of central haemomodynamics from abrachial cuff in a community setting. BMC Cardiovasc Disord. (2012) 12:48. doi: 10.1186/1471-2261-12-48

18. Protogerou AD, Argyris A, Nasothimiou E, Vrachatis D, Papaioannou TG, Tzamouranis D, et al. Feasibility and reproducibility of noninvasive 24-h ambulatory aortic blood pressure monitoring with a brachial cuffbased oscillometric device. Am J Hypertens. (2012) 25:876-82. doi: 10.1038/ajh.2012.63

19. Marwick TH, Gillebert TC, Aurigemma G, Chirinos J, Derumeaux G, Galderisi M, et al. Recommendations on the use of echocardiography in adult hypertension: a report from the European Association of Cardiovascular Imaging (EACVI) and the American Society of Echocardiography (ASE). J Am Soc Echocardiogr. (2015) 28:727-54. doi: 10.1016/j.echo.2015.05.002

20. Ma YC, Zuo L, Chen JH, Luo Q, Yu XQ, Li Y, et al. Modified glomerular filtration rate estimating equation for Chinese patients with chronic kidney disease. J Am Soc Nephrol. (2006) 17:2937-44. doi: 10.1681/ASN.20060 40368

21. Greene T, Bourgoignie JJ, Habwe V, Kusek JW, Snetselaar LG, Soucie JM, et al. Baseline characteristics in the modification of diet in Renal Disease Study. $J$ Am Soc Nephrol. (1993) 4:1221-36. doi: 10.1681/ASN.V451221
22. Mancia G, Fagard R, Narkiewicz K, Redón J, Zanchetti A, Böhm M, et al. 2013 ESH/ESC guidelines for the management of arterial hypertension. the task force for the management of arterial hypertension of the European Society of Hypertension (ESH) and of the European Society of Cardiology (ESC). $J$ Hypertens. (2013) 31:1281-357. doi: 10.1097/01.hjh.0000431740.32696.cc

23. Blood Pressure Lowering Treatment Trialists' Collaboration. Effects of different blood-pressure-lowering regimens on major cardiovascular events: results of prospectively-designed overviews of randomised trials. Lancet. (2003) 362:1527-735. doi: 10.1016/S0140-6736(03)14739-3

24. O'Brien E. 24-h ambulatory blood pressure measurement in clinical practice and research: a critical review of a technique in need of implementation. J Int Med. (2011) 269:478-95. doi: 10.1111/j.1365-2796.2011.02356.x

25. O’Brien E, Parati G, Stergiou G, Asmar R, Beilin L, Bilo G, et al. European society of hypertension working group on blood pressure monitoring. European Society of Hypertension position paper on ambulatory blood pressure monitoring. J Hypertens. (2013) 31:1731-68. doi: 10.1097/HJH.0000000000000017

26. Sega R, Facchetti R, Bombelli M, Cesana G, Corrao G, Grassi G, et al. Prognostic value of ambulatory and home blood pressures compared with office blood pressure in the general population. follow-up results from the Pressioni Arteriose Monitorate e Loro Associazioni(PAMELA) study. Circulation. (2005) 111:1777-83. doi: 10.1161/01.CIR.0000160923.04524.5B

27. Dolan E, Stanton A, Thijs L, Hinedi K, Atkins N, McClory S, et al. Superiority of ambulatory over clinic blood pressure measurement in predicting mortality: the Dublin outcome study. Hypertension. (2005) 46:15661. doi: 10.1161/01.HYP.0000170138.56903.7a

28. De la Sierra A, Banegas JR, Segura J, Segura J, Gorostidi M, Ruilope LM, et al. Ambulatory blood pressure monitoring and development of cardiovascular events in high-risk patients included in the Spanish ABPM Registry. $J$ Hypertens. (2012) 30:713-9. doi: 10.1097/HJH.0b013e328350bb40

29. Kong MG, Kim HL, Kim MA, Kim M, Park SM, Yoon HJ, et al. Relationships between blood pressure measurements and target organ damage: data from the Korea women's chest pain registry. J Clin Hypertens. (2018) 20:172430. doi: $10.1111 /$ jch. 13417

30. Jokiniitty JM, Majahalme SK, Kähönen MA, Tuomisto MT, Turjanmaa VM. Pulse pressure is the best predictor of future left ventricular mass and change in left ventricular mass: 10 years of follow-up. J Hypertens. (2001) 19:204754. doi: 10.1097/00004872-200111000-00016

31. Cirillo M, Stellato D, Laurenzi M, Panarelli W, Zanchetti A, De Santo NG. Pulse pressure and isolated systolic hypertension: association with microalbuminuria. the GUBBIO Study Collaborative Research Group. Kidney Int. (2000) 58:1211-8. doi: 10.1046/j.1523-1755.2000.00276.x

32. Cui H, Wang F, Fan L, Hu YX, Hu GL, Liu L, et al. Association factors of target organ damage: analysis of 17,682 elderly hypertensive patients in China. Chin Med J. (2011) 124:3676-81.

33. Covic A, Goldsmith DJ, Panaghiu L, Covic M, Sedor J. Analysis of the effect of hemodialysis on peripheral and central arterial pressure waveforms. Kidney Int. (2000) 57:2634-43. doi: 10.1046/j.1523-1755.2000.00124.x

34. Kollias A, Lagou S, Zeniodi ME, Boubouchairopoulou N, Stergiou GS. Association of central versus brachial blood pressure with target-organ damage. systematic review and meta-analysis. Hypertension. (2016) 67:18390. doi: 10.1161/HYPERTENSIONAHA.115.06066

35. Roman MJ, Devereux RB, Kizer JR, Lee ET, Galloway JM, Ali T, et al. Central pressure more strongly relates to vascular disease and outcome than does brachial pressure: the Strong Heart study. Hypertension. (2007) 50:197203. doi: 10.1161/HYPERTENSIONAHA.107.089078

36. Williams B, Lacy PS, Thom SM, Cruickshank K, Stanton A, Collier $D$, et al. Differential impact of blood pressure-lowering drugs on centralaortic pressure and clinical outcomes: principal results of the ConduitArtery Function Evaluation (CAFE) study. Circulation. (2006) 113:1213-25. doi: 10.1161/CIRCULATIONAHA.105.595496

37. Mitchell GF, Hwang SJ, Vasan RS, Larson MG, Pencina MJ, Hamburg NM, et al. Arterial stiffness and cardiovascular events: the Framingham Heart study. Circulation. (2010) 121:50511. doi: 10.1161/CIRCULATIONAHA.109.886655

38. Vlachopoulos C, Aznaouridis K, O’Rourke MF, Safar ME, Baou K, Stefanadis C, et al. Prediction of cardiovascular events and all-cause mortality with central haemodynamics: a systematic review and meta-analysis. Eur Heart J. (2010) 31:1865-71. doi: 10.1093/eurheartj/ehq024 
39. de la Sierra A, Pareja J, Fernández-Llama P, Armario P, Yun S, Acosta E, et al. Twenty-four-hour central blood pressure is not better associated with hypertensive target organ damage than 24-h peripheral blood pressure. J Hypertens. (2017) 35:2000-5. doi: 10.1097/HJH.0000000000 001431

40. Joint Committee for Guideline Revision. 2018 Chinese guidelines for prevention and treatment of hypertension-a report of the revision committee of Chinese guidelines for prevention and treatment of hypertension. J Geriatr Cardiol. (2019) 16:182-241. doi: 10.11909/j.issn.1671-5411.2019.03.014

41. Muiesan ML, Pasini G, Salvetti M, Calebich S, Zulli R, Castellano M, et al. Cardiac and vascular structural changes. prevalence and relation to ambulatory blood pressure in a middle-aged general population in northern Italy: the Vobarno Study. Hypertension. (1996) 27:104652. doi: 10.1161/01.HYP.27.5.1046

42. Mancia G, Parati G, Hennig M, Flatau B, Omboni S, Glavina F, et al. Relation between blood pressure variability and carotid artery damage in hypertension: baseline data from the European Lacidipine Study on Atherosclerosis (ELSA). J Hypertens. (2001) 19:1981-9. doi: 10.1097/00004872-200111000-00008

43. Fouad DA, Al Araby HH, Ashraf M, El-Kousy AE. Comparison between central and ambulatory blood pressure measurements in early detection of end organ damage: a single-center prospective non-randomized controlled trial. Egypt Heart J. (2019) 71:14. doi: 10.1186/s43044-019-0 013-3
44. Bai B, Teliewubai J, Lu Y, Yu S, Xiong J, Chi C, et al. Comparison of pulse wave velocity and pulse pressure amplification in association with target organ damage in community-dwelling elderly: The Northern Shanghai Study. Hypertens Res. (2018) 41:372-81. doi: 10.1038/s41440-018-0027-3

Conflict of Interest: The authors declare that the research was conducted in the absence of any commercial or financial relationships that could be construed as a potential conflict of interest.

Publisher's Note: All claims expressed in this article are solely those of the authors and do not necessarily represent those of their affiliated organizations, or those of the publisher, the editors and the reviewers. Any product that may be evaluated in this article, or claim that may be made by its manufacturer, is not guaranteed or endorsed by the publisher.

Copyright $\odot 2022 \mathrm{Hu}$, Zhao, Wang, Chao, Tang, Cheng, Tan, Butlin, Avolio, Wang and Zuo. This is an open-access article distributed under the terms of the Creative Commons Attribution License (CC BY). The use, distribution or reproduction in other forums is permitted, provided the original author(s) and the copyright owner(s) are credited and that the original publication in this journal is cited, in accordance with accepted academic practice. No use, distribution or reproduction is permitted which does not comply with these terms. 